\title{
Shear-Induced Topology Changes in Liquid Crystals of the Soybean Lecithin/DDAB/Water System
}

\author{
Gemma Montalvo*\# ${ }^{\#}$ Mercedes Valiente , and Ali Khan ${ }^{\S}$
}

SUPPORTING INFORMATION AVAILABLE 
Table 1: Detailed composition of the lamellar liquid crystalline samples.

\begin{tabular}{|c|c|c|c|}
\hline Lecithin (wt\%) & DDAB (wt \%) & $\chi_{\mathbf{D}}=\mathrm{n}_{\mathrm{D}} /\left(\mathrm{n}_{\mathrm{L}}+\mathrm{n}_{\mathrm{D})}\right.$ & $\mathbf{R}=\left(\mathrm{n}_{\mathrm{L}}+\mathrm{n}_{\mathrm{D}}\right) / \mathrm{n}_{\mathrm{W}}$ \\
\hline \multicolumn{4}{|c|}{ Lecithin/DDAB = 1 (at wt \%) Series 1} \\
\hline 25 & 25 & 0.626 & 0.0311 \\
\hline 30 & 30 & 0.626 & 0.0466 \\
\hline 40 & 40 & 0.626 & 0.1244 \\
\hline \multicolumn{4}{|c|}{ Lecithin/DDAB = 0.2 (at wt \%) Series 3} \\
\hline 2 & 10 & 0.893 & 0.0050 \\
\hline 3 & 15 & 0.893 & 0.0080 \\
\hline 4 & 20 & 0.893 & 0.0115 \\
\hline \multicolumn{4}{|c|}{ Lecithin/water = 1 (at wt \%) Series 4} \\
\hline 48 & 4 & 0.122 & 0.0265 \\
\hline 41 & 17 & 0.409 & 0.0385 \\
\hline 38 & 25 & 0.524 & 0.0502 \\
\hline 30 & 40 & 0.690 & 0.0752 \\
\hline 23 & 55 & 0.800 & 0.1216 \\
\hline 19 & 62 & 0.845 & 0.1502 \\
\hline \multicolumn{4}{|c|}{ Lecithin/water $=0.5$ (at wt\%) Series 5} \\
\hline
\end{tabular}




\begin{tabular}{|c|c|c|c|}
\hline 34 & 3 & 0.128 & 0.0144 \\
\hline 28 & 17 & 0.504 & 0.0239 \\
\hline 25 & 25 & 0.626 & 0.0311 \\
\hline 21 & 40 & 0.761 & 0.0524 \\
\hline 13 & 62 & 0.889 & 0.1086 \\
\hline 8 & 73 & 0.938 & 0.1593 \\
\hline \multicolumn{4}{|c|}{$20 \mathrm{wt} \%$ water Series 6} \\
\hline 75 & 5 & 0.100 & 0.0970 \\
\hline 40 & 40 & 0.626 & 0.1244 \\
\hline 23 & 55 & 0.800 & 0.1216 \\
\hline 19 & 62 & 0.845 & 0.1502 \\
\hline \multicolumn{4}{|c|}{40 wt $\%$ water Series 7} \\
\hline 55 & 5 & 0.132 & 0.0369 \\
\hline 41 & 18 & 0.423 & 0.0404 \\
\hline 38 & 25 & 0.524 & 0.0502 \\
\hline 30 & 30 & 0.626 & 0.0466 \\
\hline 21 & 40 & 0.761 & 0.0524 \\
\hline 10 & 50 & 0.893 & 0.0545 \\
\hline
\end{tabular}




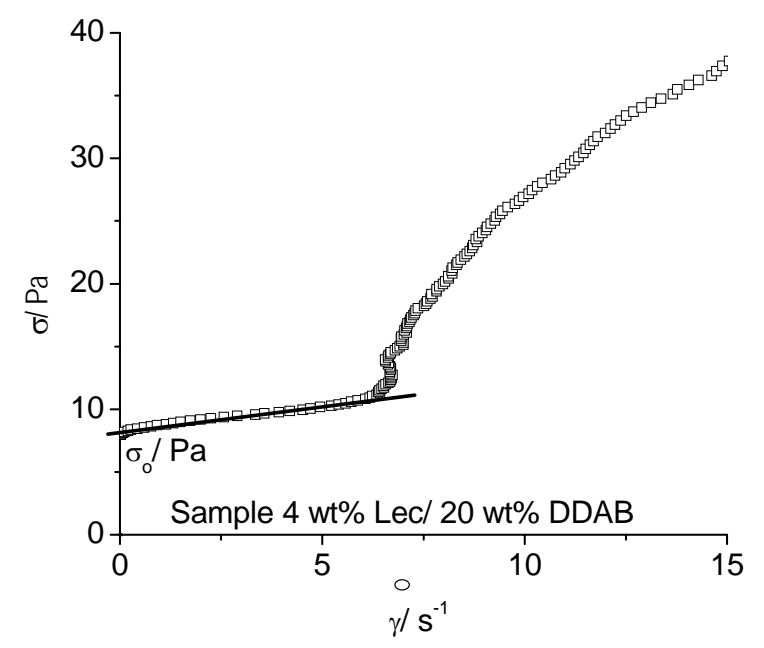

Figure i). Yield-stress determination.

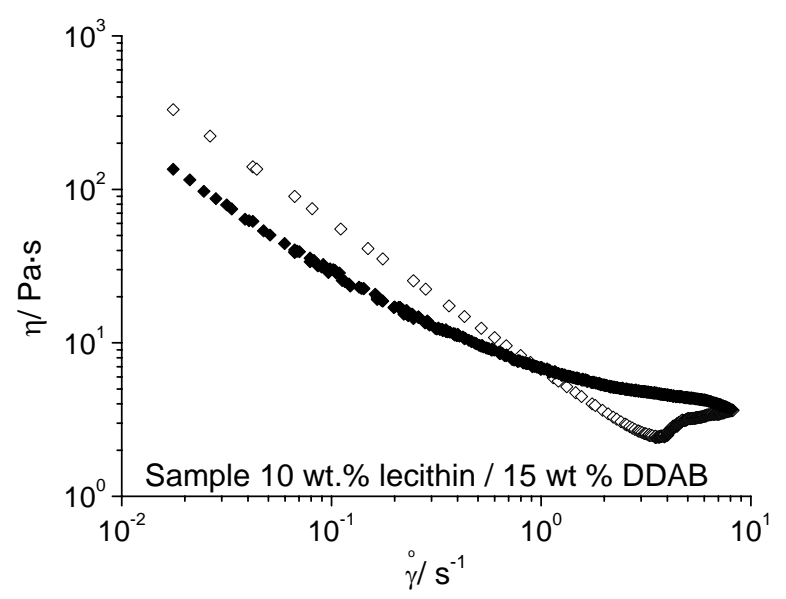

Figure ii). Viscosity as a function of shear rate in a cycle of increasing (open symbols) and decreasing stresses (closed symbols), for the swollen lamellar phase. Same behaviour was obtained for the collapsed lamellar samples in the DDAB/water ( $w \mathrm{t} \%)<1$ region. 


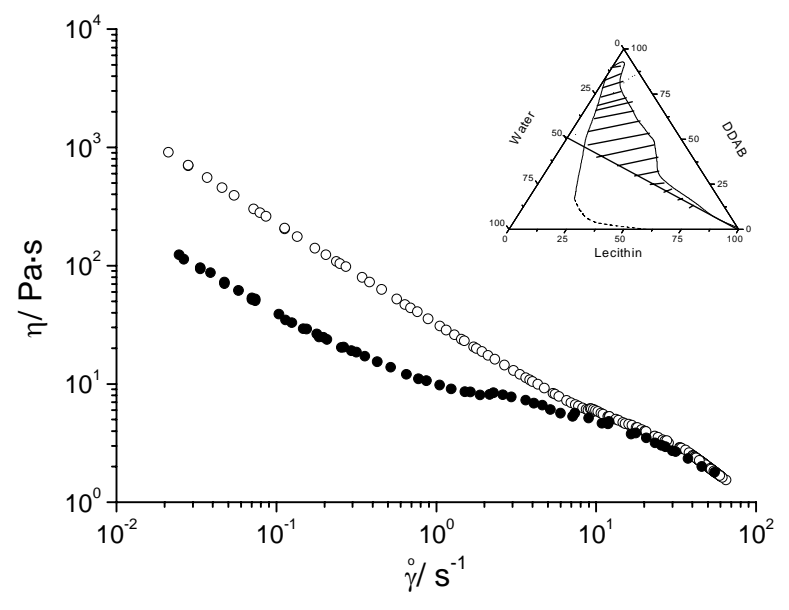

Figure iii). Representative profile of viscosity as a function of shear rate in exponentially increasing (open symbols) and decreasing (closed symbols) applied stresses. Insert: The dash-line represents $\mathrm{DDAB} /$ water $(\mathrm{wt} \%)=1$; the shaded area indicates samples with a power-law flow curve for the collapsed lamellar samples. 